\title{
Design of Non-contact Coordinate Measurement System
}

\author{
Jingliang Liu, a , Chunchao Pang ${ }^{2, b}$, Jianguo Fang ${ }^{3, c}$ \\ ${ }^{1,2,3}$ Aviation Key Laboratory of Science and Technology on Precision Manufacturing, \\ NanYuan East Road NO.5, FengTai District, BeiJing, China \\ aJL7519970@163.com, bPcc-hit@163.com, cfkylek@sina.com.
}

Keywords: Non-contact measurement, synchronized measurement data, Optical probe

Abstract. In this paper, several technical problems were conquered, such as synchronous collecting measurement data, calibrating the beam's space position of optical probe, and unified coordinate system,ect. Finally, the non-contact coordinate measuring system was built by using Pearl1298 CMM and optical probe produced by Micro-Epsilon. What is more, the accuracy of the system can meet precision measuring with the accurate experiment on measuring the standard ball.

\section{Introduction}

Currently, $90 \%$ of CMMs use contact probes in high precision measurement from home and abroad. Although it has the properties of high precision, high maturity, and so on, it has low detection efficiency, especially to detect larger workpieces or complex curved surface parts. With the development of optical technology, the optical non-contact measurement system gets more and more attentions, and the accuracy is becoming higher and higher. Some factories have designed their own non-contact measurement system, as Wenzel, Nextec, Hexgon, and Renishaw, etc. Some product's measure accuracy is up to $0.01 \mathrm{~mm}$. It is wrong to compare non-contact measuring system with contact measuring system about their precisions, but non-contact CMM system can also do precision measurement job now and there is a great room for improvement. ${ }^{[4]}$

Technologies about non-contact coordinate measurement system are still in infancy, the key technologies have not been resolved before 2012. Based on the support of Aviation Innovation Fund, the Beijing Precision Engineering Institute for Aircraft Industry has developed non-contact coordinate measurement system with conquering the key technologies. The measure system not only has high precision on testing workpieces, but also shortens the gap with foreign countries in the field of non-contact coordinate measuring technology.

\section{Establishing Non-contact coordinate measurement system}

There are two important parts in non-contact measurement system. One is Pearl 1298 CMM which was produced by one of departments of our factory, and it has the properties of advanced mechanical design, excellent dynamic performance, flexible work space of $1200 \times 900 \times 800 \mathrm{~mm}$, and the measurement uncertainty $2.5 \mathrm{um}$. The other is ILD1700-20 optical probe which was produced by micro-epsilon, and its measurement range is from 40 to $60 \mathrm{~mm}$.

Non-contact coordinate measurement is equipped with optical probe perfectly with the help of transforming the PH10M's extension bar. At the same time, the optical probe has many measurement angles when measure some object because the PH10M has two axes named A axis and B axis. The optical probe is shown in Fig.1(a), and the non-contact coordinate system is shown in Fig.1(b). ${ }^{[2,3]}$ 


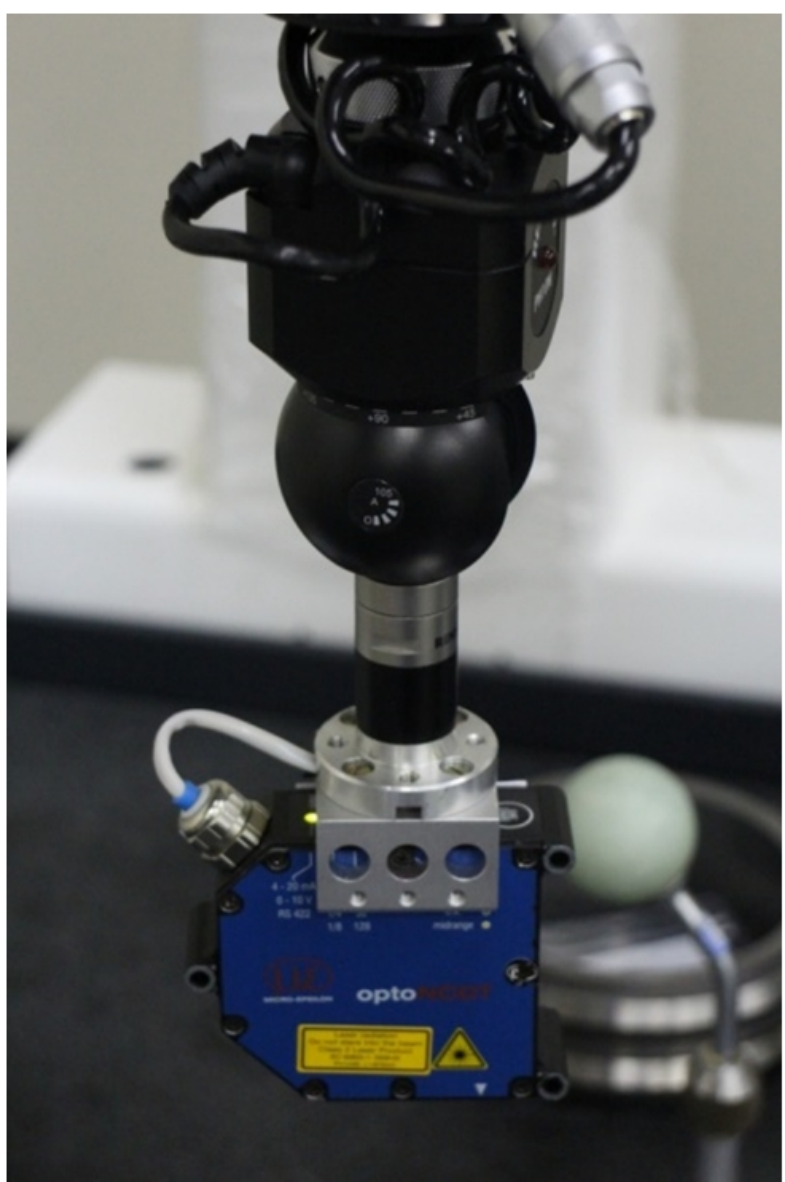

Fig. 1(a)

Optical probe system

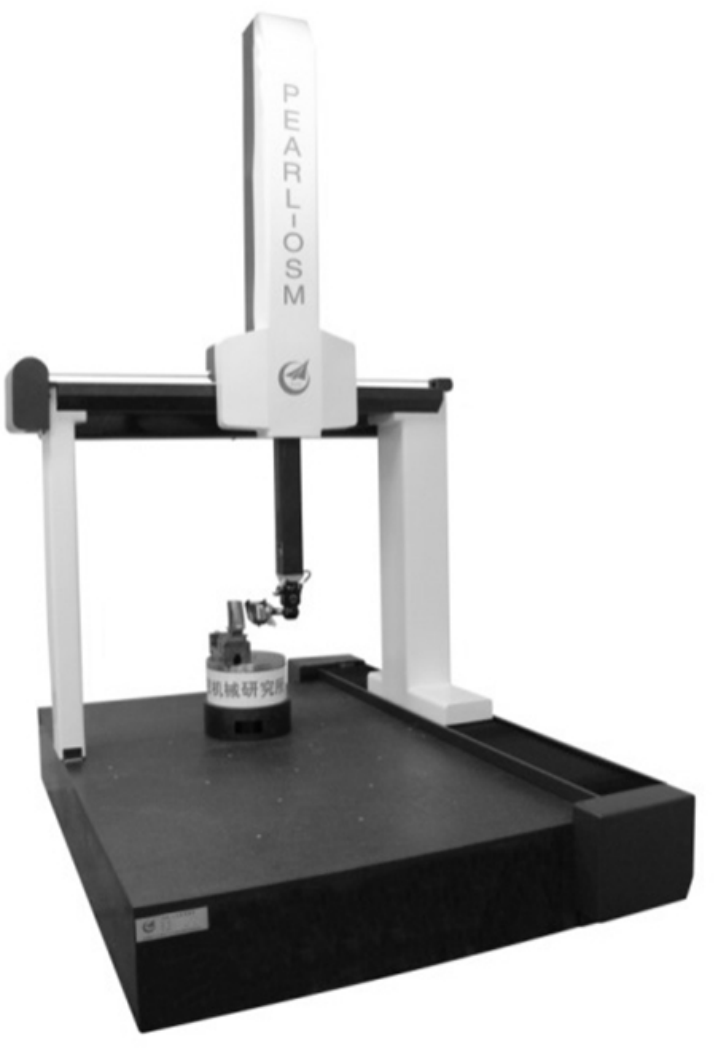

Fig. 1(b)

Non-contact coordinate measurement system

\section{The Key Technologies of the Measurement system}

\section{Synchronous measurement data acquisition technology}

Synchronizing the measurement data between CMM and optical probe is one of the key technologies when the system is scanning workpiece. It is necessary to guarantee the synchronous of the measurement data to obtain high precision measurement. After many schemes of synchronization experiments, the external pulse mode is used for synchronizing measurement data, and the accuracy of synchronization is under $\pm 4 \mathrm{um}$, so the system could meet the requirment of the precision measurement. The scheme of synchronization is shown in Fig. 2 .

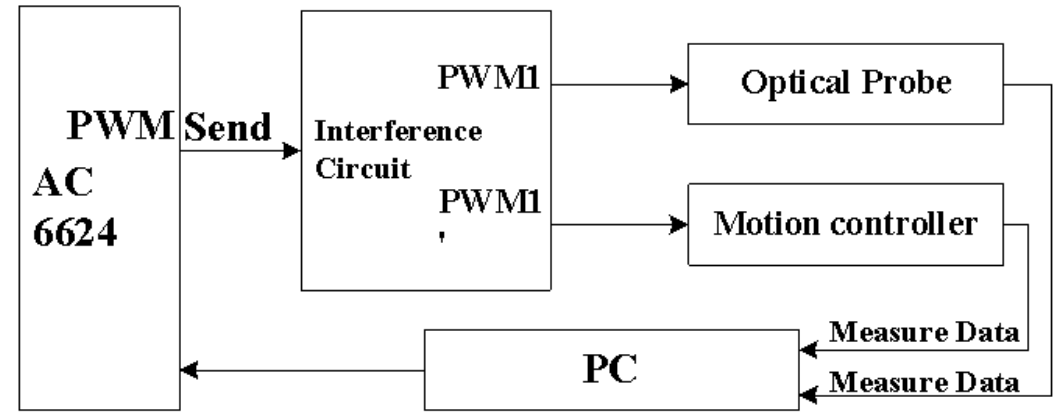

Fig.2 The scheme of synchronization

\section{Calibrating the beam's space position of optical probe}

Regardless of which kind of probe equipped on the CMM, it is very necessary to calibrate the probe. The non-contact coordinate measurement system used the "The Calibration Method by Seven Points ${ }^{[1]}$, to calibrate the optical probe's position. Complex mathematical model was bulit to achieve the auto-calibration process. The auto-calibration flowchart is shown in Fig. 3: 


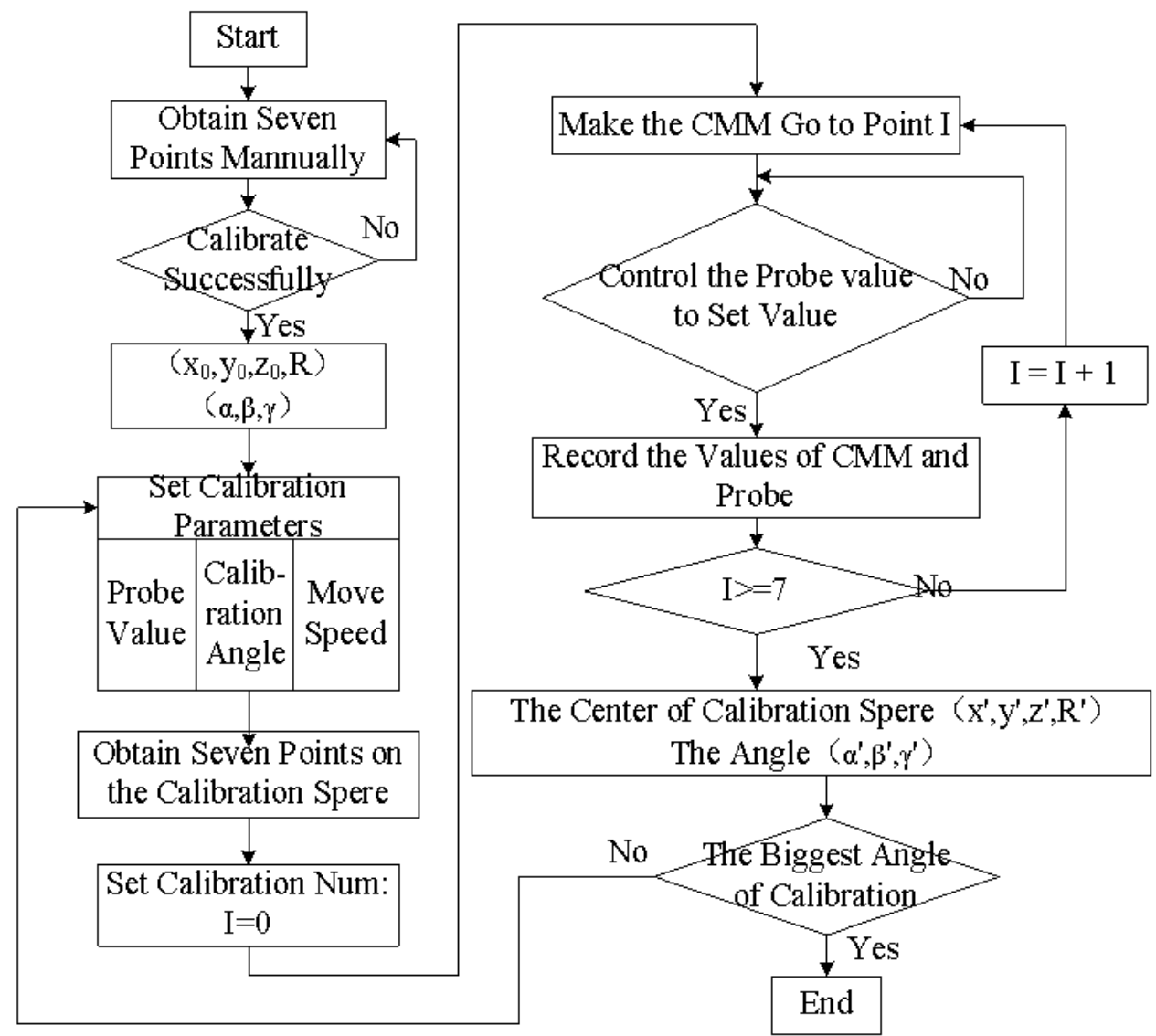

Fig.3 The auto-calibration process

\section{Coordinate system transformation}

In most case, no element of workpiece can be measured with single probe angle. Another probe angle is needed, and then, the coordinate transformation needs to be solved. The measured data in the same coordinate system makes the measurement meaning. The measured data under other angles should be transformed to the $\left(\mathrm{A} 0{ }^{\circ}, \mathrm{B} 0^{\circ}\right)$. The process is as follow:

First, calibrating $\left(\mathrm{A} 0{ }^{\circ}, \mathrm{B} 0^{\circ}\right)$ probe angle, the center of the standard ball $(\mathrm{X} 0, \mathrm{Y} 0, \mathrm{Z} 0)$ and the actual angle of probe are collected.

Then, calibrating $\left(\mathrm{A} 0{ }^{\circ}, \mathrm{B} 0^{\circ}\right)$ probe angle using the same standard ball, the sphere center $(\mathrm{Xa}, \mathrm{Ya}$

, Za) and the actual angle of probe are collected.

Finally, measured results $\left(\mathrm{X}_{1}\right.$ ', $\left.\mathrm{Y}_{1}{ }^{\prime}, \mathrm{Z}_{1}{ }^{\prime}\right)$ under an angle of (Aa,Bb) should be transformed into measured value $\left(\mathrm{X}_{1}, \mathrm{Y}_{1}, \mathrm{Z}_{1}\right)$ under the angle of $\left(\mathrm{A} 00^{\circ}, \mathrm{B} 0^{\circ}\right)$ by using the formula (1).

$$
\begin{aligned}
& X_{1}=X_{1}{ }^{\prime}+X_{0}-X a \\
& Y_{1}=Y_{1}{ }^{\circ}+Y_{0}-Y a \\
& Z_{1}=Z_{1}{ }^{\prime}+Z_{0}-Z a
\end{aligned}
$$

\section{Accuracy test}

After establishing the non-contact coordinate system, the accuracy of the system needs to be verified. The accuracy experiment process is shown as follows: Firstly, calibrating the optical probe's position and angle; Secondly, putting the double ball bar on the platform which is shown in Fig.4, then measure the two balls by the system under the situation of the distance among 49 to 51; Thirdly, analyzing the measured result. The result of experiment is in following table. 


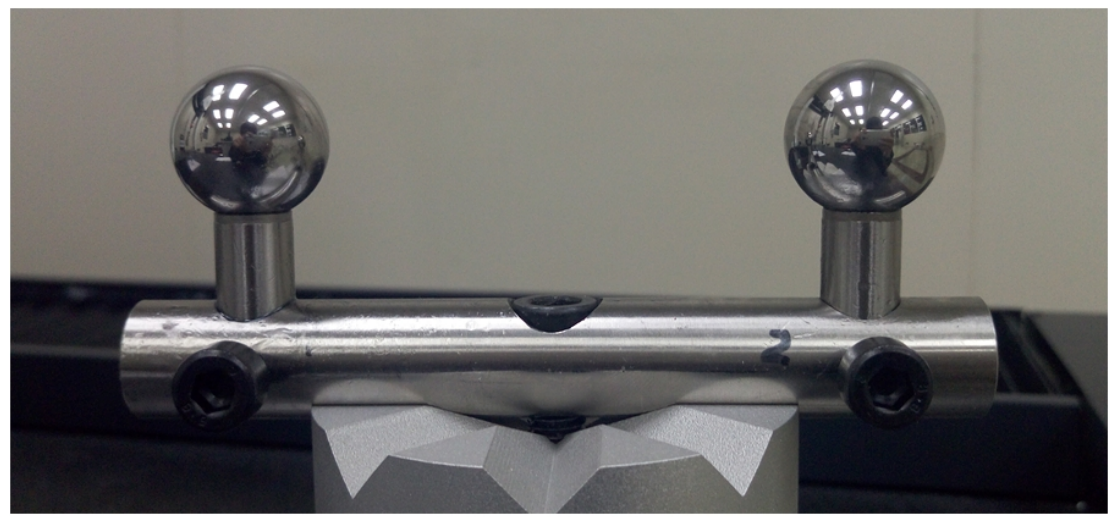

Fig 4 Double ball bar

Table 1.Experiment results:

\begin{tabular}{ccccc}
\hline Sort Order & $1^{\text {st }}$ Experiment & $2^{\text {nd }}$ Experiment & $3^{\text {rd }}$ Experiment & $\begin{array}{c}\text { Nominal } \\
\text { value }\end{array}$ \\
\hline $\begin{array}{c}1^{\text {st }} \text { Sphere's } \\
\text { Diameter }\end{array}$ & 23.993 & 23.996 & 23.999 & 23.996 \\
\hline $\begin{array}{c}2^{\text {nd }} \text { Sphere's } \\
\text { Diameter }\end{array}$ & 23.995 & 23.992 & 24.001 & 23.998 \\
\hline $\begin{array}{c}\text { Distance of } \\
\text { Sphere's Center }\end{array}$ & 100.059 & 100.057 & 100.063 & 100.061 \\
\hline
\end{tabular}

From the Table1, the conclusion can be draw that the non-contact measurement system can meet high precision measurement.

\section{Summary}

Based on solving synchronous measured data, calibrating the optical probe's position and beam angle, unified coordinate system, etc, the non-contact measurement system was finally established. After precision test, the system can do high measurement job. The next step is to improve system stability.

\section{References}

[1]Jianguo Fang, Tan zhang, Liang Fang. A Calibration Method of the Spot-Structured optical probe's spatial position and vector. China, 201310493454.2.

[2] H Kwan Lee, Hyun-Pung Park. Automated inspection planning of free-form shape parts by laser scanning [J], Robotics and Computer Integrated Manufacturing, 2000, 16(65):201-210.

[3] Information on http:www.micro-epsilon.com

[4]Guoxiong Zhang. The Development of Coordinate Measurement Machine[J]. Infrared and laser engineering, 2008,37(S1): 1-5.

[5]Zexiao Xie , The Calibration method of no-contact probe based on Vision. China , 200510044577.3[P].2006-03-01. 